\title{
Eco-evolutionary dynamics in freshwater systems
}

\author{
Luc De MEESTER*, Jelena PANTEL \\ Laboratory of Ecology, Evolution and Conservation, University of Leuven, Ch. Deberiotstraat 32, 3000 Leuven, Belgium \\ *Corresponding author: luc.demeester@bio.kuleuven.be
}

\begin{abstract}
There is an increasing recognition that evolutionary dynamics may occur at timescales that are sufficiently rapid to impact ecological responses to environmental change and influence ecological dynamics. In the development of the emerging paradigm of eco-evolutionary dynamics, studies of freshwater organisms have been very important. Here we highlight a number of key observations and achievements, and point to remaining challenges.
\end{abstract}

Key words: eco-evolutionary feedbacks, evolving metacommunities, rapid evolution, population dynamics, community ecology, aquatic systems.

Received: July 2013. Accepted: October 2013.

\section{ECO-EVOLUTIONARY DYNAMICS}

Ecological and evolutionary processes have largely been studied separately, yet growing evidence suggests that ecological and evolutionary dynamics can occur at the same temporal and spatial scales (Hendry and Kinnison, 1999; Hairston et al., 2005). This implies that there is potential for a wide range of interactions between ecological and evolutionary processes (Hairston et al., 2005; Thompson 2005; Schoener 2011). Evolutionary change may potentially influence population (Pelletier et al., 2009), community (Urban et al., 2008) and ecosystem dynamics (Fussmann et al., 2007; Matthews et al., 2011). If eco-evolutionary interactions are widespread under natural conditions, they may fundamentally alter the way populations, communities and ecosystems respond to environmental gradients and change (Fussmann et al., 2007; Urban et al., 2008; Pelletier et al., 2009). Ignoring these interactions can distort our predictions of biological responses to human impact, such as fisheries (Olsen et al., 2004), land use and urbanization (Cheptou et al., 2008), and climate change (De Meester et al., 2011; Urban et al., 2012). For instance, urbanization drives seed trait changes in plants (Cheptou et al., 2008), which may feedback, through their effect on dispersal, on metapopulation and metacommunity structure of plant species at a regional level. Eco-evolutionary dynamics may often be important in the case of anthropogenic change, as it has been claimed that eco-evolutionary dynamics will be especially important in the case of strong selection pressures and extreme environments (Hanski 2012).

The field of eco-evolutionary dynamics integrates insights from ecology and evolution and may fundamentally change our understanding of ecological and evolutionary processes. A prerequisite in the study of eco-evolutionary dynamics is that both ecological as well as evolutionary processes are considered. Eco-evolutionary interactions imply that ecological processes influence evolutionary change, evolutionary change impacts ecological processes, or both, and that these interactions happen at overlapping time scales. Evidence for evolution occurring on timescales potentially overlapping with ecological processes has increased steadily in the past 20 years (Thompson, 1998; Hendry and Kinnison, 1999) and has contributed greatly to the development of the concept of eco-evolutionary feedbacks. This research direction has precedent in studies of character displacement (Aarssen, 1983; Chesson 2000; Schluter 2000), colonization dynamics (Roughgarden, 1972; Parsons, 1983) and stress ecology (Antonovics, 1972). However, proof of the principle that genotypic identity strongly impacts ecological dynamics (Yoshida et al., 2003; De Meester et al., 2007; Fukami et al., 2007; Venail et al., 2008; Harmon et al., 2009) and that these changes can occur over short time spans (Hairston et al., 2005; Becks et al., 2012) have only relatively recently inspired the idea that eco-evolutionary dynamics may be common and may strongly impact the ecological dynamics we observe in nature. Understanding the impact of eco-evolutionary dynamics may be crucial to better understand both ecological and evolutionary dynamics.

We refer to eco-evolutionary feedbacks if both directions of the interaction, ecology influencing evolution and evolution influencing ecology, are studied and observed in the same system. Here one can differentiate between a very strict definition of a feedback, where the evolutionary change in the trait value feeds back on the same ecological process that caused the trait to change (e.g., predation impacting the evolution of a defence trait that immediately impacts predation rates), or a more relaxed definition where the feedback can also involve other eco- 
logical processes (e.g., predation impacting the evolution of a defence trait that impacts competitive strength or host-parasite dynamics). We here use the relaxed definition, referring to any feedback between ecological and evolutionary processes. There are still relatively few studies that considered the complete feedback loop of ecological processes leading to evolution and the resulting changes feeding back to ecological processes. A strong example is given by the evolution of grazing resistance in the alga Chlamydomonas caused by changes in predator (rotifer) densities impacting subsequent rotifer dynamics (Becks et al., 2012). Many studies rather provide proof of concept for parts of the feedback process (De Meester $e t$ al., 2007; Harmon et al., 2009) or focus on the impact of phenotypic trait variation without explicitly considering genotypic trait variation (Palkovacs and Post 2009; Walsh et al., 2012). These latter studies certainly provide convincing evidence for the potential of strong eco-evolutionary feedbacks, but rely on the assumption, often substantiated through references to heritabilities of the traits studied as quantified by other studies, that the phenotypic differences are linked to genetic differences. Similarly, studies quantifying only the effect of genotype identity on ecological dynamics provide strong indications for the potential impact of eco-evolutionary feedbacks, but rely on the assumption that the differentiated genotypes used in the experiments are the product of recent evolution. For instance, De Meester et al. (2007) showed that genotype identity in the water flea Daphnia magna can influence community assembly in zooplankton. While the genotypes used in this experiment were isolated from neighbouring populations that were connected until a few years before the study, this study did not document the full process of genetic differentiation in the two ponds and its feedback on community composition.

Evidence that eco-evolutionary dynamics may be widespread may lie in the fact that they have been considered and quantified in studies from several ecological fields. Eco-evolutionary dynamics are observed in evolutionary demography, as evolutionary change in trait values alters fitness, which feeds back on population dynamics (Pelletier et al., 2007; Ozgul et al., 2009; Ozgul et al., 2010; Smallegange and Coulson 2013). Similarly, ecoevolutionary dynamics have been considered extensively in studies of host-parasite and other co-evolutionary dynamics (Thompson, 2005). For example, genotype identity and associated virulence and resistance impacts disease spread, population dynamics and epidemiology (Duffy et al., 2009). These examples indicate that the recognition of dynamic interactions between ecological and evolutionary processes and their importance is not new. However, one should not derive from this that ecoevolutionary dynamics is just a new term to refer to obvious and widely recognized dynamics. The field incites so much enthusiasm because eco-evolutionary dynamics can explain substantial portions of biological systems that the study of ecology or evolution in isolation cannot, and this increased understanding has important practical benefits, for instance in predicting changes in community and ecosystem properties or responses to anthropogenic environmental change.

\section{ECO-EVOLUTIONARY DYNAMICS: AQUATIC SYSTEMS AS MODELS}

While powerful studies of eco-evolutionary dynamics have been carried out on terrestrial (Crutsinger et al., 2008; Poisot et al., 2011; Agrawal et al., 2013) and marine (Reusch et al., 2005) systems, it is striking that many studies of eco-evolutionary dynamics and feedbacks have been carried out using freshwater systems (see further). We see several reasons for this. First, freshwater systems are clearly delineated habitats in a terrestrial matrix, which makes it easy to determine the boundaries of populations and determine connectivity among populations. This is a major advantage of ponds and lakes (De Meester et al., 2005) but also holds for rivers, where connectivity is high within but low among river catchments and species such as fish are bound by direct hydrological connections. Most studies of eco-evolutionary dynamics in freshwater systems indeed either focus on lake and pond populations or on lakes or pools that are interconnected by rivers. Three-spine sticklebacks independently colonizing lakes in northern Canada and showing parallel radiations (Barrett et al., 2008; Jones et al., 2012), alewives in landlocked or connected lakes (Palkovacs and Post 2009), and guppies in pools of tropical streams (Bassar et al., 2012) are all examples of river-connected study systems featured in eco-evolutionary research. In addition to offering well-delineated habitat patches in a hostile matrix, the differences in ecological conditions among even neighbouring aquatic habitats are often strong, such as when habitats differ in the presence of dispersal-limited predators (Reznick et al., 1990; Orsini et al., 2012).

Eco-evolutionary impacts of (genetic) intraspecific variation on ecological dynamics are clearly not restricted to freshwater systems, as local adaptation similarly occurs in terrestrial and marine habitats. However, clearly delineated habitats such as ponds and lakes, river systems, tidal pools, isolated rocky outcrops, forest fragments, etc. provide appropriate sampling units. Many aquatic species are also excellent candidates for studying eco-evolutionary feedbacks for the same reasons they are model ecological or evolutionary organisms - ease of culture, short generation times, small body sizes enabling the establishment of large populations in controlled experiments, and reproduction cycles that allow working with clonal lineages. These features apply to the water flea Daphnia (Miner et al., 2012), rotifers (Becks et al., 2012) and protists (Bell, 
2013). Essential features of aquatic systems, especially ponds, are also relatively easy to capture in cattle tanks and mesocosms, which enables replicated controlled experiments under semi-natural conditions (Spivak et al., 2010; Logue et al., 2011). Mesocosm studies can incorporate complexities such as multiple trophic levels within relatively small systems (Verreydt et al., 2012). This in principle allows one to carry out experiments offering different conditions in mesocosms to quantify evolution in these systems, and to subsequently measure the impact of this evolution on ecological interactions (Matthews et al., 2011). Finally, aquatic organisms with sufficiently small body sizes and fast generation times can also be cultured under highly standardized constant conditions using chemostats (Yoshida et al., 2003; Becks et al., 2012). This is a powerful approach to create constant environmental conditions that allow very controlled selection pressures and detailed monitoring of population dynamics, the versatility of which is being increasingly explored in powerful studies aimed at detecting the mechanisms underlying eco-evolutionary feedbacks (Becks et al., 2012). Another interesting feature of aquatic systems that has yet to be exploited in the framework of eco-evolutionary dynamics is the possibility to reconstruct evolutionary dynamics from layered dormant egg banks (Jeppesen et al., 2001; Orsini et al., 2013). In systems with a well-documented history of ecological change and associated evolutionary dynamics (Cousyn et al., 2001; Hairston et al., 2001) it should be possible to also quantify the impact of the observed evolutionary changes on ecological processes.

\section{CASE STUDIES}

We next highlight the results of selected studies that explored eco-evolutionary dynamics in inland aquatic systems. These examples illustrate interesting features and knowledge gaps while also addressing the potential importance of eco-evolutionary dynamics and how eco-evolutionary dynamics can be studied using different approaches and model systems. This overview is not intended to be complete but rather to inspire further reading, as there are an increasing number of exciting studies of an increasing variety of systems to be discovered.

\section{Rotifer-algae predator-prey dynamics}

Some of the best-developed studies of eco-evolutionary dynamics involve unicellular algae, Chlorella vulgaris and Chlamydomonas reinhardtii, and one of their predators, the rotifer Brachionus calyciflorus. Using C. vulgaris, Yoshida et al. (2003) showed that the predator-prey cycles in chemostats differed substantially depending on whether genetically uniform or genetically diverse algae were present. Uniform cultures led to short predator-prey cycles and a typical quarter-phase time lag between prey and predator peak densities, while much longer, out-ofphase cycles were observed, and predator and prey peak lags nearly out of phase were observed with genetically diverse cultures. These results agreed with predictions of a model that incorporated evolution of anti-predator traits in the algae, but could not be explained by non-evolutionary models (Shertzer et al., 2002; Yoshida et al., 2007).

These studies are complemented by the study of Becks et al. (2012), which used a different algal species, C. reinhardtii. This species demonstrated similar population dynamic patterns in the presence of $B$. calyciflorus and, unlike $C$. vulgaris, possesses a heritable defence trait (clump formation, which reduces the effectiveness of gape-limited predation) that can be tracked visually. The complete genome sequence available for $C$. reinhardtii also permitted tracking suites of genes whose expression changed in concert with the degree of cell clumping. Becks et al. (2012) clearly linked population cycles in the presence of rotifers to fluctuating selection for cell clumping and to corresponding changes in expression of genes associated with defence. Trait evolution followed the dynamics expected from models that explicitly considered evolution. Following the methods developed by Ellner et al. (2011), they also compared the relative influence of evolving algal clump formation and ecological changes in algal density on rotifer population dynamics and found their influence was of a similar magnitude and that evolutionary dynamics were antagonistic to ecological dynamics, thus buffering overall fluctuations in population densities (Becks et al., 2012). This latter result is important, as it suggests that eco-evolutionary dynamics may often be important in reducing rather than enhancing ecological dynamics, and may thus be important in systems where one would not expect them because the observed dynamics in population size or community composition are minor. Finally, Becks et al. (2012) also used transcriptomics to link genotypic trait values in antipredator defence to gene regulation. Interestingly, while they detected typical cycles in gene expression, these were not repeatable at the gene level, indicating that phenotypic trait changes in different cycles may be mediated by different combinations of genes. This observation cautions against overly optimistic embracing of genomic tools as an easy replacement of quantitative genetics. In this study, the results were much more repeatable and easy to interpret at the level of genotypic trait values than at the level of gene expression.

While this system produced arguably one of the best empirical studies of eco-evolutionary dynamics so far, with a thorough understanding of both dynamics and mechanisms, some gaps remain. Chemostats are very controlled and simplified systems that deviate strongly from the complexities in the natural world, both in terms of species richness as well as in the complexity and variation 
in environmental conditions. One critical extension would be to test the impact of evolutionary dynamics in a community setting, where the algae consist of different species with unique defence mechanisms. Algae differing in defence traits would probably experience differential changes in relative abundances due to predation. It would be very revealing if one could document to what extent under these conditions evolutionary dynamics would still have an important impact on predator-prey dynamics (de Mazancourt et al., 2008). Establishing evidence in more diverse communities will be crucial in showing that ecoevolutionary dynamics may be important in well-established natural settings beyond the colonization phase. In a recent paper, Hultinen et al. (2013) report the population dynamics in chemostats with two predators and one prey species. This paper did not explore the impact of evolution, but illustrates that complexity rapidly increases when intraguild predation is included in the system.

\section{Daphnia genotype identity and community assembly}

De Meester et al. (2007) tested whether establishment success of an invading zooplankton community in a Daphnia magna population differs depending on the genetic identity of the resident $D$. magna populations. They inoculated D. magna genotypes from two ecologically different ponds at identical densities and genetic diversity (eight clones) into mesocosms and let them establish a stable population before inoculating a species rich zooplankton community and monitoring establishment success of these immigrants during four consecutive weeks. They found that establishment success of immigrant zooplankton differed strongly among treatments with the two different population origins of the D. magna clones. Pantel et al. (2011) obtained complementary results using different $D$. pulex-pulicaria populations, finding differential success of populations invading mesocosms with established zooplankton communities. Both these studies thus provide strong empirical support for community assembly being impacted by genetic identity. Similar proof of concept has been obtained in studies of plant communities (Vellend, 2006; Crutsinger et al., 2008). All of these studies, however, demonstrate that intraspecific genetic variation impacts community structure but not that the differences in genotype identity were the result of recent evolution. These authors thus used existing intraspecific standing genetic variation rather than evolutionary dynamics to show that evolution might impact community attributes. There is much evidence for rapid evolution in Daphnia (Miner et al., 2012) and in the case of De Meester et al. (2007) the two populations from which the genotypes in the experiment were isolated were connected until a few years before the experiment. This strengthens the indications of a potential effect of rapid local adaptation on community assembly. Yet, at this stage, these stud- ies only provide direct evidence for the evolution-to-ecology part of the feedback loop.

\section{Stream guppies and ecosystem dynamics}

Studies on the Trinidadian guppy, Poecilia reticulata, and the stream ecosystem they inhabit hint at a range of factors possibly driving eco-evolutionary dynamics. By combining mathematical models, stream flow-through mesocosms, and in situ manipulations of stream pools, a strong influence of guppy phenotype on ecosystem processes could be demonstrated and important advances were made towards clarifying the mechanism of this influence (Palkovacs et al., 2009; Bassar et al., 2010; Bassar et al., 2012). In experimental streams, the effect of guppy phenotype on factors such as primary productivity, decomposition, and nutrient flux was as large or larger than the effect of guppy invasion or of doubling guppy density (Palkovacs et al., 2009; Bassar et al., 2010). Bassar et al. (2012) also evaluated the effect of both direct pathways, such as invertebrate consumption, and indirect pathways, such as loss of invertebrate nutrient input via excretion, on eco-evolutionary dynamics and found that indirect effects of differing magnitude mediated the effect of guppy phenotype on stream ecosystems. Another remarkable study simultaneously evaluated the effects of guppy invasion, guppy phenotype, and guppy coevolution (Palkovacs et al., 2009). Killifish-guppy coevolution (the addition of guppy in experimental streams with killifish from the same or different native streams) affected total invertebrate biomass and decomposition rates more than the ecological process of simply adding guppies to streams, suggesting that the source of invading guppies may be much more important for stream ecosystems than their invasion.

\section{Cascades of eco-evolutionary dynamics}

Trophic cascades are important in aquatic ecosystems (Pace et al., 1999), and one may thus expect phenotypic variation in a top predator to mediate eco-evolutionary dynamics at lower trophic levels. Landlocked alewife (Alosa pseudoharengus) populations independently evolved multiple times from a core ancestral anadromous population (Palkovacs et al., 2008) and their phenotypic differences have been linked to heritable life history variation among associated lake Daphnia ambigua populations (Walsh and Post, 2011, 2012). A subsequent study (Walsh et al., 2012) showed that faster growth rates of Daphnia from lakes with anadromous alewives impacted algal development and thus potential ecosystem characteristics. This study nicely illustrates the potentially strong impact of phenotypic differences in top predators cascading down to biomass of primary producers and ecosystem characteristics such as net primary production. It evaluates the impact of intraspe- 
cific phenotypic variation, as the link between phenotypic and genetic differences in alewife populations is indirect. Also, while the authors provide evidence for parallels between their experimental results and the dynamics of zooand phytoplankton in lakes without alewives, with landlocked alewives and with anadromous alewives, strong conclusions are difficult as this comparison assumes that the studied lakes only differ in phenotype of alewives. The occurrence of anadromous alewife populations differs across lake types, so it is unclear to what extent the differences observed among lakes result from timing of alewife predation pressure or from the phenotypic differences among the populations (Walsh et al., 2012). Despite these limitations, this case study thus far provides the strongest evidence for the potential that evolution of trait values in specific species impacts eco-evolutionary dynamics at multiple trophic levels.

One very important argument for the importance of ecoevolutionary dynamics is the commonality of adaptive genetic polymorphism and local adaptation. Populations have been shown to genetically track environmental changes within short time spans from months to years. Rapid adaptive evolution has been reported in experimental evolution trials using algae (Bell 2013) and in selection experiments on sticklebacks (Barrett et al., 2008), has been documented from local genetic adaptation in pigmentation in Asellus aquaticus (Hargeby et al., 2005; Eroukhmanoff et al., 2009), and is a recurrent observation in studies of the water flea Daphnia, e.g. with respect to thermal adaptation (Van Doorslaer et al., 2009a; Van Doorslaer et al., 2010), resistance to pollution (Jansen et al., 2010; Jansen et al., 2011), salinity (Latta et al., 2012), UV tolerance (Miner and Kerr, 2011), parasites (Ebert, 2005; Decaestecker et al., 2007), and predation (Cousyn et al., 2001; Fisk et al., 2007, Latta et al., 2007). Van Doorslaer et al. (2009b) showed that rapid genetic adaptation reduced establishment success of preadapted, immigrant genotypes, suggesting an eco-evolutionary feedback loop that can potentially affect genetic structure of the species at the regional scale.

\section{CHALLENGES}

The above case studies show that eco-evolutionary dynamics are potentially a strong structuring factor of population, community and ecosystem attributes and dynamics. Each of these case studies has strengths and weaknesses, but collectively they provide strong proof of principle that eco-evolutionary dynamics should not be ignored in our efforts to explain the dynamics and characteristics of natural systems. They also show that it is difficult to capture in one study both the complexity of the full eco-evolutionary feedback and its dynamics, and that often shortcuts are used - using ecotypes or genetically distant lineages drawn from existing standing genetic variation at the population or regional level, or focusing on phenotypic differences. The latter approach is, however, risky, because phenotypic differences may be mediated by phenotypic plasticity, causing incorrect inference of evolutionary dynamics.

The overall message from this brief account is that there is ample evidence for evolutionary changes occurring in the same time span as ecological change and that the potential of these evolutionary changes to feedback on the dynamics and nature of ecological processes is substantial. We have related proof of principle for feedbacks at the level of interactions among species and the resulting population dynamics, community composition and ecosystem characteristics. There is, in other words, much potential in natural systems for eco-evolutionary dynamics. However, one key challenge that remains is learning the degree to which eco-evolutionary dynamics impact the patterns observed in nature. How much insight in patterns and dynamics of populations, communities and ecosystems do we gain by incorporating eco-evolutionary dynamics? There is a strong need for studies that assess the relative importance of ecological and evolutionary dynamics in nature and relate this to regional and local species diversity, as well as to landscape, environmental and species characteristics. To advance both fundamental and applied science, it is critical to develop approaches that combine ecological and evolutionary processes across natural landscapes. In addition to studying the impact of eco-evolutionary dynamics in natural landscapes, experimental studies must also increase the ecological complexity considered in their design, to obtain insight on whether this reduces or increases the importance of evolutionary dynamics. The field of eco-evolutionary dynamics will also benefit from studies that link genes and gene expression to genotypic trait values. We believe there are exciting times ahead as these perspectives are realized and feel that aquatic systems and model species are likely to play a significant role in this realization.

\section{ACKNOWLEDGMENTS}

We gratefully thank KU Leuven Research Fund for their support of the Centre of Excellence in "Eco- and Socio-evolutionary dynamics" (PF/2010/007); this work was also financially supported by FWO projects G. 0468.10 and G.0614.11.

\section{REFERENCES}

Aarssen LW, 1983. Ecological combining ability and competitive combining ability in plants - toward a general evolutionary-theory of coexistence in systems of competition. Am. Nat. 122:707-731.

Agrawal AA, Johnson MTJ, Hastings AP, Maron JL, 2013. A field experiment demonstrating plant life-history evolution and its eco-evolutionary feedback to seed predator populations. Am. Nat. 181:S35-S45. 
Antonovics J, 1972. Population dynamics of grass Anthoxanthum odoratum on a zinc mine. J. Ecol. 60:351-365.

Barrett RDH, Rogers SM, Schluter D, 2008. Natural selection on a major armor gene in threespine stickleback. Science 322:255-257.

Bassar RD, Ferriere R, Lopez-Sepulcre A, Marshall MC, Travis J, Pringle CM, Reznick DN, 2012. Direct and Indirect ecosystem effects of evolutionary adaptation in the Trinidadian guppy (Poecilia reticulata). Am. Nat. 180:167-185.

Bassar RD, Marshall MC, López-Sepulcre A, Zandonà E, Auer SK, Travis J, Pringle CM, Flecker AS, Thomas SA, Fraser DF, Reznick DN, 2010. Local adaptation in Trinidadian guppies alters ecosystem processes. P. Natl. Acad. Sci. USA 107:3616-3621.

Becks L, Ellner SP, Jones LJ, Hairston NG, 2012. The functional genomics of an eco-evolutionary feedback loop: linking gene expression, trait evolution, and community dynamics. Ecol. Lett. 15:492-501.

Bell G, 2013. Evolutionary rescue of a green alga kept in the dark. Biol. Lett. 9:20120823.

Cheptou PO, Carrue O, Rouifed S, Cantarel A, 2008. Rapid evolution of seed dispersal in an urban environment in the weed Crepis sancta. P. Natl. Acad. Sci. USA 105:3796-3799.

Chesson P, 2000. Mechanisms of maintenance of species diversity. Annu. Rev. Ecol. Syst. 31:343-366.

Cousyn C, De Meester L, Colbourne JK, Brendonck L, Verschuren D, Volckaert F, 2001. Rapid, local adaptation of zooplankton behavior to changes in predation pressure in the absence of neutral genetic changes. P. Natl. Acad. Sci. USA 98:6256-6260.

Crutsinger GM, Souza L, Sanders NJ, 2008. Intraspecific diversity and dominant genotypes resist plant invasions. Ecol. Lett. 11:16-23.

de Mazancourt C, Johnson E, Barraclough TG, 2008. Biodiversity inhibits species' evolutionary responses to changing environments. Ecol. Lett. 11:380-388.

De Meester L, Declerck S, Stoks R, Louette G, Van de Meutter F, De Bie T, Michels E, Brendonck L, 2005. Ponds and pools as model systems in conservation biology, ecology and evolutionary biology. Aquat. Conserv. 15:715-725.

De Meester L, Louette G, Duvivier C, Van Darnme C, Michels E, 2007. Genetic composition of resident populations influences establishment success of immigrant species. Oecologia 153:431-440.

De Meester L, Van Doorslaer W, Geerts A,Orsini L, Stoks R, 2011. Thermal genetic adaptation in the water flea daphnia and its impact: an evolving metacommunity approach. Integr. Comp. Biol. 51:703-718.

Decaestecker E, Gaba S, Raeymaekers JAM, Stoks R, Van Kerckhoven L, Ebert D, De Meester L, 2007. Host-parasite 'Red Queen' dynamics archived in pond sediment. Nature 450:870-873.

Duffy MA, Hall SR, Caceres CE, Ives AR, 2009. Rapid evolution, seasonality, and the termination of parasite epidemics. Ecology 90:1441-1448.

Ebert D, 2005. Ecology, epidemiology, and evolution of parasitism in Daphnia [Internet]. Available from: http://www.ncbi.nlm.nih.gov/entrez/query.fcgi?db=Books

Ellner SP, Geber MA, Hairston NG, 2011. Does rapid evolution matter? Measuring the rate of contemporary evolution and its impacts on ecological dynamics. Ecol. Lett. 14:603-614.

Eroukhmanoff F, Hargeby A, Svensson EI, 2009. Rapid adaptive divergence between ecotypes of an aquatic isopod inferred from F-ST-Q(ST) analysis. Mol. Ecol. 18:4912-4923.

Fisk DL, Latta LC, Knapp RA, Pfrender ME, 2007. Rapid evolution in response to introduced predators I: rates and patterns of morphological and life-history trait divergence. BMC Evol. Biol. 7:22.

Fukami T, Beaumont HJE, Zhang XX, Rainey PB. 2007. Immigration history controls diversification in experimental adaptive radiation. Nature 446:436-439.

Fussmann GF, Loreau M, Abrams PA, 2007. Eco-evolutionary dynamics of communities and ecosystems. Funct. Ecol. 21:465-477.

Hairston NG, Ellner SP, Geber MA, Yoshida T, Fox JA, 2005. Rapid evolution and the convergence of ecological and evolutionary time. Ecol. Lett. 8:1114-1127.

Hairston NG, Holtmeier CL, Lampert W, Weider LJ, Post DM, Fischer JM, Caceres CE, Fox JA, Gaedke U, 2001. Natural selection for grazer resistance to toxic cyanobacteria: evolution of phenotypic plasticity? Evolution 55:2203-2214.

Hanski I, 2012. Eco-evolutionary dynamics in a changing world. Ann. N.Y. Acad. Sci. 1249:1-17.

Hargeby A, Stoltz J, Johansson J, 2005. Locally differentiated cryptic pigmentation in the freshwater isopod Asellus aquaticus. J. Evol. Biol. 18:713-721.

Harmon LJ, Matthews B, Des Roches S, Chase JM, Shurin JB, Schluter D, 2009. Evolutionary diversification in stickleback affects ecosystem functioning. Nature 458:1167-1170.

Hendry AP, Kinnison MT, 1999. Perspective: the pace of modern life: measuring rates of contemporary microevolution. Evolution 53:1637-1653.

Hiltunen T, Jones LE, Ellner SP, Hairston NGJ, 2013. Temporal dynamics of a simple community with intraguild predation: an experimental test. Ecology 94:773-779.

Jansen M, Stoks R, Coors A, van Doorslaer W, de Meester L, 2011. Collateral damage: rapid exposure-induced evolution of pesticide resistance leads to increased susceptibility to parasites. Evolution 65:2681-2691.

Jansen M, Stoks R, Decaestecker E, Coors A, Van de Meutter F, De Meester L, 2010. Local exposure shapes spatial patterns in infectivity and community structure of Daphnia parasites. J. Anim. Ecol. 79:1023-1033.

Jeppesen E, Leavitt P, De Meester L, Jensen JP, 2001. Functional ecology and palaeolimnology: using cladoceran remains to reconstruct anthropogenic impact. Trends Ecol. Evol. 16:191-198.

Jones FC, Grabherr MG, Chan YF, Russell P, Mauceli E, Johnson J, Swofford R, Pirun M, Zody MC, White S, Birney E, Searle S, Schmutz J, Grimwood J, Dickson MC, Myers RM, Miller CT, Summers BR, Knecht AK, Brady SD, Zhang HL, Pollen AA, Howes T, Amemiya C, Broad Institute Genome Sequencing Platform and Whole Genome Assembly Team, Lander ES, Di Palma F, Lindblad-Toh K, Kingsley DM, 2012. The genomic basis of adaptive evolution in threespine sticklebacks. Nature 484:55-61.

Latta LC, Bakelar JW, Knapp RA, Pfrender ME, 2007. Rapid evolution in response to introduced predators II: the contribution of adaptive plasticity. BMC Evol. Biol. 7:21.

Latta LC, Weider LJ, Colbourne JK, Pfrender ME, 2012. The 
evolution of salinity tolerance in Daphnia: a functional genomics approach. Ecol. Lett. 15:794-802.

Logue JB, Mouquet N, Peter H, Hillebrand H, The Metacommunity Working Group, 2011. Empirical approaches to metacommunities: a review and comparison with theory. Trends Ecol. Evol. 26:482-491.

Matthews B, Narwani A, Hausch S, Nonaka E, Peter H, Yamamichi M, Sullam KE, Bird KC, Thomas MK, Hanley TC, Turner CB, 2011. Toward an integration of evolutionary biology and ecosystem science. Ecol. Lett. 14:690-701.

Miner BE, De Meester L, Pfrender ME, Lampert W, Hairston NG, 2012. Linking genes to communities and ecosystems: Daphnia as an ecogenomic model. P. R. Soc. B 279:18731882.

Miner BE, Kerr B, 2011. Adaptation to local ultraviolet radiation conditions among neighbouring Daphnia populations. P. R. Soc. B 278:1306-1313.

Olsen EM, Heino M, Lilly GR, Morgan MJ, Brattey J, Ernande B, Dieckmann U, 2004. Maturation trends indicative of rapid evolution preceded the collapse of northern cod. Nature 428:932-935.

Orsini L, Schwenk K, De Meester L, Colbourne JK, Pfrender ME, Weider LJ, 2013. The evolutionary time machine: using dormant propagules to forecast how populations can adapt to changing environments. Trends Ecol. Evol. 28:274-282.

Orsini L, Spanier KI, De Meester L, 2012. Genomic signature of natural and anthropogenic stress in wild populations of the waterflea Daphnia magna: validation in space, time and experimental evolution. Mol. Ecol. 21:2160-2175.

Ozgul A, Childs DZ, Oli MK, Armitage KB, Blumstein DT, Olson LE, Tuljapurkar S, Coulson T, 2010. Coupled dynamics of body mass and population growth in response to environmental change. Nature 466:482-U485.

Ozgul A, Tuljapurkar S, Benton TG, Pemberton JM, CluttonBrock TH, Coulson T, 2009. The dynamics of phenotypic change and the shrinking sheep of St. Kilda. Science 325:464-467.

Pace ML, Cole JJ, Carpenter SR, Kitchell JF, 1999. Trophic cascades revealed in diverse ecosystems. Trends Ecol. Evol. 14:483-488.

Palkovacs EP, Marshall MC, Lamphere BA, Lynch BR, Weese DJ, Fraser DF, Reznick DN, Pringle CM, Kinnison MT, 2009. Experimental evaluation of evolution and coevolution as agents of ecosystem change in Trinidadian streams. Philos. T. Roy Soc. B 364:1617-1628.

Palkovacs EP, Post DM, 2009. Experimental evidence that phenotypic divergence in predators drives community divergence in prey. Ecology 90:300-305.

Pantel JH, Leibold MA, Juenger TE, 2011. Population Differentiation in Daphnia Alters Community Assembly in Experimental Ponds. Am. Nat. 177:314-322.

Parsons PA, 1983. The evolutionary biology of colonizing species. Cambridge University Press: 262 pp.

Pelletier F, Clutton-Brock T, Pemberton J, Tuljapurkar S, Coulson T, 2007. The evolutionary demography of ecological change: linking trait variation and population growth. Science 315:1571-1574.

Pelletier F, Garant D, Hendry AP, 2009. Eco-evolutionary dynamics. Philos. T. R. Soc. B 364:1483-1489.

Poisot T, Bever JD, Nemri A, Thrall PH, Hochberg ME, 2011.
A conceptual framework for the evolution of ecological specialisation. Ecol. Lett. 14:841-851.

Reusch TBH, Ehlers A, Hammerli A, Worm B, 2005. Ecosystem recovery after climatic extremes enhanced by genotypic diversity. P. Natl. Acad. Sci. USA 102:2826-2831.

Reznick DA, Bryga H, Endler JA, 1990. Experimentally induced life-history evolution in a natural population. Nature 346:357-359.

Roughgarden J, 1972. Evolution of niche width. Am. Nat. 106:683-718.

Schluter D, 2000. The ecology of adaptive radiation. Oxford University Press: 296 pp.

Schoener TW, 2011. The newest synthesis: understanding the interplay of evolutionary and ecological dynamics. Science 331:426-429.

Shertzer KW, Ellner SP, Fussmann GF, Hairston NG, 2002. Predator-prey cycles in an aquatic microcosm: testing hypotheses of mechanism. J. Anim. Ecol. 71:802-815.

Smallegange IM, Coulson T, 2013. Towards a general, population-level understanding of eco-evolutionary change. Trends Ecol. Evol. 28:143-148.

Spivak AC, Vanni MJ, Mette EM, 2010. Moving on up: can results from simple aquatic mesocosm experiments be applied across broad spatial scales? Freshwater Biol. 56:279-291.

Thompson JN, 1998. Rapid evolution as an ecological process. Trends Ecol. Evol. 13:329-332.

Thompson JN, 2005. The geographic mosaic of coevolution. University of Chicago Press: 400 pp.

Urban MC, De Meester L, Vellend M, Stoks R, Vanoverbeke J, 2012. A crucial step toward realism: responses to climate change from an evolving metacommunity perspective. Evol. Appl. 5:154-167.

Urban MC, Leibold MA, Amarasekare P, De Meester L, Gomulkiewicz R, Hochberg ME, Klausmeier CA, Loeuille N, de Mazancourt C, Norberg J, Pantel JH, Strauss SY, Vellend M, Wade MJ, 2008. The evolutionary ecology of metacommunities. Trends Ecol. Evol. 23:311-317.

Van Doorslaer W, Stoks R, Duvivier A, Bednarska, De Meester L, 2009a. Population dynamics determine genetic adaptation to temperature in Daphnia. Evolution 63:1867-1878.

Van Doorslaer W, Stoks R, Swillen I, Feuchtmayr H, Atkinson D, Moss B, De Meester L, 2010. Experimental thermal microevolution in community-embedded Daphnia populations. Clim. Res. 43:81-89.

Van Doorslaer W, Vanoverbeke J, Duvivier C, Rousseaux S, Jansen M, Jansen B, Feuchtmayr H, Atkinson D, Moss B, Stoks R, De Meester L, 2009b. Local adaptation to higher temperatures reduces immigration success of genotypes from a warmer region in the water flea Daphnia. Glob. Change Biol. 15:3046-3055.

Vellend M, 2006. The consequences of genetic diversity in competitive communities. Ecology 87:304-311.

Venail PA, MacLean, Bouvier T, Brockhurst MA, Hochberg ME, Mouquet N, 2008. Diversity and productivity peak at intermediate dispersal rate in evolving metacommunities. Nature 452:210-U257.

Verreydt D, De Meester L, Decaestecker E, Villena MJ, Van der Gucht K, Vannormelingen P, Vyverman W, Declerck SAJ, 2012. Dispersal-mediated trophic interactions can generate apparent patterns of dispersal limitation in aquatic metacommunities. Ecol. Lett. 15:218-226. 
Walsh MR, DeLong JP, Hanley TC, Post DM, 2012. A cascade of evolutionary change alters consumer-resource dynamics and ecosystem function. P. Roy. Soc. B-Biol. Sci. 279:31843192.

Walsh MR, Post DM, 2011. Interpopulation variation in a fish predator drives evolutionary divergence in prey in lakes. P. Roy. Soc. B-Biol. Sci. 278:2628-2637.

Walsh MR, Post DM, 2012. The impact of intraspecific variation in a fish predator on the evolution of phenotypic plasticity and investment in sex in Daphnia ambigua. J. Evol. Biol. 25:80-89.

Yoshida T, Ellner SP, Jones LE, Bohannan BJM, Lenski RE, Hairston NG, 2007. Cryptic population dynamics: Rapid evolution masks trophic interactions. Plos Biology 5:18681879.

Yoshida T, Jones LE, Ellner SP, Fussmann GF, Hairston NG, 2003. Rapid evolution drives ecological dynamics in a predator-prey system. Nature 424:303-306. 